\title{
Colgajo dorsal ancho preservador de músculo para la reconstrucción de defectos en la extremidad inferior en niños
}

\author{
Muscle-sparing latissimus dorsi flap for inferior limb's defects reconstruction in children
}

Mauricio M. García-Pérez, Jorge T. Palacios-Zertuche, Fernando M. Martínez-Ruiz y

Gabriel A. Mecott-Rivera*

Servicio de Cirugía Plástica Estética y Reconstructiva, Hospital Universitario Dr. José Eleuterio González, Universidad Autónoma de Nuevo León, Monterrey, Nuevo León, México

\section{Resumen}

Objetivo: Evaluar los resultados estéticos y funcionales, así como el grado de satisfacción, posteriores a la reconstrucción de defectos de la extremidad inferior en niños con colgajo de dorsal ancho preservador del músculo como una alternativa para estos casos. Método: Se incluyeron pacientes menores de 18 años con defectos en la extremidad inferior tratados en un periodo de 3 años. Se realizó colgajo dorsal ancho preservador de músculo en todos los pacientes. Se valoraron la apariencia estética, la fuerza muscular del área donadora y la función en el área receptora a los 3 meses de realizado el colgajo. Resultados: Se realizaron 13 colgajos de dorsal ancho preservadores de músculo. La apariencia estética de las áreas donadora y receptora fue satisfactoria en la mayoría de los casos. La mayoría de los pacientes presentaron una fuerza muscular normal (M5) en el sitio donador y pudieron deambular. Conclusiones: El colgajo de dorsal ancho preservador de músculo tiene un grado de satisfacción muy elevado, excelente simetría y preservación de la función muscular del sitio donador. Es una técnica ideal en defectos tisulares importantes de la extremidad inferior en la población pediátrica.

Palabras clave: Colgajo. Reconstrucción. Extremidad inferior. Niños.

\begin{abstract}
Objective: To evaluate the aesthetic and functional outcomes as well as the degree of postoperative satisfaction of the lower limb's defects reconstruction with a muscle-sparing latissimus dorsi flap in children in order to propose this kind of reconstruction as a secure alternative. Method: In this study were included patients under 18 years with lower limb's defects treated over a period of 3 years. A muscle-sparing latissimus dorsi flaps was performed in all patients. After three months the flap was performed, it was evaluated the donor's area muscular strength, recipient area function and aesthetic appearance. Results: They were made 13 muscle-sparing latissimus dorsi flaps. In most cases, the donor and recipient area has satisfactory aesthetic appearance, most of the patients had a normal muscular strength (M5) in donors area and were able to walk. Conclusions: The reconstruction using a muscle-sparing latissimus dorsi flap has a very high level of satisfaction, excellent symmetry, and preservation of donor area muscle function. It is an ideal technique for major tissue defects of lower limb in children.
\end{abstract}

Key words: Flap. Reconstruction. Lower limb. Children.

\section{Correspondencia:}

*Gabriel A. Mecott-Rivera

Avda. Francisco I. Madero y Gonzalitos s/n

Col. Mitras Centro

Fecha de recepción: 24-04-2020

C.P. 64460 , Monterrey, N.L., México

E-mail: gabriel.mecottrv@uanl.edu.mx
Cir Cir. 2021;89(3):361-368

Contents available at PubMed DOI: $10.24875 / C I R U .20000389$
www.cirugiaycirujanos.com (http://creativecommons.org/licenses/by-nc-nd/4.0/). 


\section{Introducción}

El trauma grave en la extremidad inferior en población pediátrica es menos frecuente que en los adultos. Las causas más comunes son por trauma de alta energía en el tobillo o el pie por accidentes de vehículo de motor, maquinaria eléctrica, heridas por arma de fuego y caídas ${ }^{1}$.

Las técnicas quirúrgicas que suelen emplearse en este sitio usualmente requieren microcirugía; sin embargo, la reconstrucción con colgajos libres en la población pediátrica presenta una serie de consideraciones especiales. Dentro de estas podemos mencionar el efecto potencial sobre el crecimiento y el desarrollo, los problemas psicológicos que conlleva el realizar una cirugía mayor en los pacientes pediátricos, la discapacidad a edades tempranas, la falta de cooperación y el menor calibre de los vasos ${ }^{1-3}$, por lo que representa un gran reto terapéutico.

No obstante, la microcirugía en esta población presenta altas tasas de éxito ${ }^{4-9}$ debido a la falta de desarrollo de la capa muscular en los vasos, lo que disminuye la presencia de espasmos y no se desarrolla aterosclerosis, lo que explica la baja incidencia de problemas vasculares ${ }^{10}$.

Los colgajos descritos con más frecuencia para la reconstrucción del miembro inferior son los de serrato anterior, recto abdominal y dorsal ancho,11,12. El colgajo de dorsal ancho es uno de los más utilizado en microcirugía ${ }^{13}$, pero presenta la desventaja de producir un déficit funcional en la extremidad superior, con pérdida de extensión, aducción y rotación interna del brazo ${ }^{14-16}$, además de las alteraciones estéticas que ocasiona en el sitio donador, como la cicatriz y el cambio de forma de la silueta torácica ${ }^{17,18}$.

Para evitar estas complicaciones, se ha descrito el uso de colgajo de dorsal ancho preservador de músculo, que a diferencia del colgajo de dorsal ancho tradicional solo incluye una porción de este múscu$10^{19-21}$. Se han reportado colgajos en los que se ha utilizado la rama interna o medial de la arteria toracodorsal para tomar un segmento muscular, los cuales han presentado excelentes resultados funcionales ${ }^{22,23}$ obteniendo un colgajo más delgado y de fácil mane$\mathrm{jo}^{24}$. Es posible realizar un colgajo preservador de músculo tanto con la rama transversa como con la descendente de la arteria toracodorsal, y se ha demostrado que no hay diferencias significativas en la perfusión musculocutánea de ambas ramas ${ }^{25}$. Este tipo de colgajos ha demostrado tener buenos resultados estéticos en cuanto a la simetría de la silueta torácica y la cicatriz del sitio donador, sin presentar déficit de la fuerza muscular ${ }^{23}$. Existen reportes de colgajos libres de dorsal ancho tradicional, sin preservación de músculo, para la reconstrucción de la extremidad inferior en niños como una buena opción para la cobertura, pero no evalúan el déficit funcional del sitio donador y tampoco los resultados estéticos que se logran con este tipo de colgajos ${ }^{1-3,6,26}$.

Existen pocos estudios sobre el uso de colgajos de dorsal ancho preservadores de músculo en pacientes pediátricos ${ }^{22,27,28}$, y los que se han realizado no incluyen solo pacientes para reconstrucción del miembro inferior. Así mismo, el análisis estético y funcional posterior a la utilización de dicho colgajo es pobre, por lo que decidimos realizar este estudio con el objetivo de evaluar el resultado estético y funcional del colgajo de dorsal ancho preservador de músculo (en los sitios receptor y donador) utilizado en la reconstrucción de defectos de la extremidad inferior en niños.

\section{Método}

Este estudio se realizó en el Hospital Universitario Dr. José Eleuterio González, en Monterrey, Nuevo León, México. Es un estudio observacional, prospectivo, descriptivo y longitudinal, que se llevó a cabo durante el periodo del 1 de marzo de 2015 al 28 de febrero de 2018.

Los criterios de inclusión fueron pacientes menores de 18 años que presentaron defectos de la extremidad inferior que se trataron dentro de los primeros 15 días después de la lesión y que requirieron cobertura con colgajo libre. Todos los pacientes ya tenían resueltas las lesiones vasculares y óseas al momento de realizarles el colgajo. Se excluyeron los pacientes con alteración del estado de conciencia o que presentaban mala calidad de los vasos receptores. El criterio de eliminación fue que, por cualquier motivo, no fuera posible el seguimiento especificado en el protocolo.

De todos los pacientes se evaluaron datos demográficos, como sexo y edad, etiología de la lesión, días transcurridos desde el ingreso hospitalario hasta la realización del colgajo, días de estancia intrahospitalaria y seguimiento.

\section{Técnica quirúrgica}

Se utilizó la técnica para el colgajo libre de dorsal ancho preservador de músculo empleando como pedículo la rama descendente de la arteria toracodorsal y 
realizando la anastomosis con microcirugía, empleando una o dos arterias perforantes. Previo al procedimiento se realiza marcaje localizando un punto a $8 \mathrm{~cm}$ por debajo del pliegue axilar posterior y $2 \mathrm{~cm}$ por detrás del borde lateral del dorsal ancho, y mediante Doppler portátil se identifican y marcan las perforantes que corresponden al trayecto de la rama descendente de la arteria toracodorsal. Posteriormente se diseña el colgajo teniendo como eje la línea media axilar.

En el quirófano se preparó el área receptora mediante desbridamiento, lo que nos dio las medidas reales del defecto a cubrir, y se identificaron los vasos receptores. Se colocaba el paciente en decúbito lateral y se dejaba expuesto el lado a procurar del colgajo.

Se realizaron la incisión y la disección en el borde lateral de la isla marcada, incluyendo el borde lateral del dorsal ancho junto con las perforantes previamente marcadas. Los límites de resección del músculo dorsal ancho que se incluyeron con la isla fueron $3 \mathrm{~cm}$ distales a la perforante de mayor flujo detectada por el Doppler y $3 \mathrm{~cm}$ mediales al borde lateral del músculo. A continuación, se disecaron los vasos proximales hasta llegar a los vasos subescapulares, preservando la integridad del nervio toracodorsal para posteriormente realizar la anastomosis terminoterminal con el vaso receptor con Ethilon® 9-0 (Figs. 1 y 2).

\section{Seguimiento}

Los pacientes se evaluaron semanalmente durante el primer mes y luego cada mes hasta los 9 meses. La evaluación estética y funcional aplicando las escalas se realizó en el tercer mes posterior al procedimiento quirúrgico en todos los pacientes.

\section{EvaluaCión ESTÉtICA}

En la visita del tercer mes posterior al procedimiento quirúrgico se valoraron la apariencia estética de las cicatrices y la simetría corporal tanto del sitio donador como del sitio receptor, además de la movilidad del brazo, en relación al remanente del dorsal ancho.

Se realizaron dos evaluaciones: una por el padre o tutor y otra por dos cirujanos por separado, uno que formaba parte del equipo de investigación y otro ciego a la investigación, por lo que se obtuvieron tres evaluaciones de cada paciente (39 evaluaciones en total). Se evaluaron las características de los sitios donador y receptor, la apariencia estética de la cicatriz en la espalda y en la extremidad inferior mediante una escala
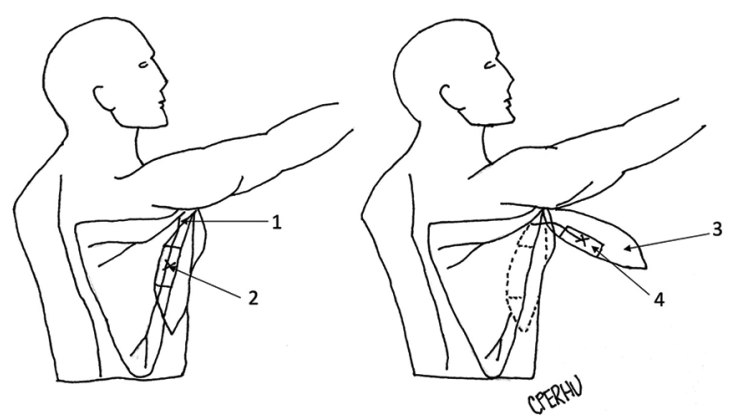

Figura 1. Diseño y levantamiento del colgajo dorsal ancho preservador de músculo. 1: rama descendente de la arteria toracodorsal; 2: arteria perforante; 3: isla de piel; 4: porción muscular del dorsal ancho incluida en el colgajo.

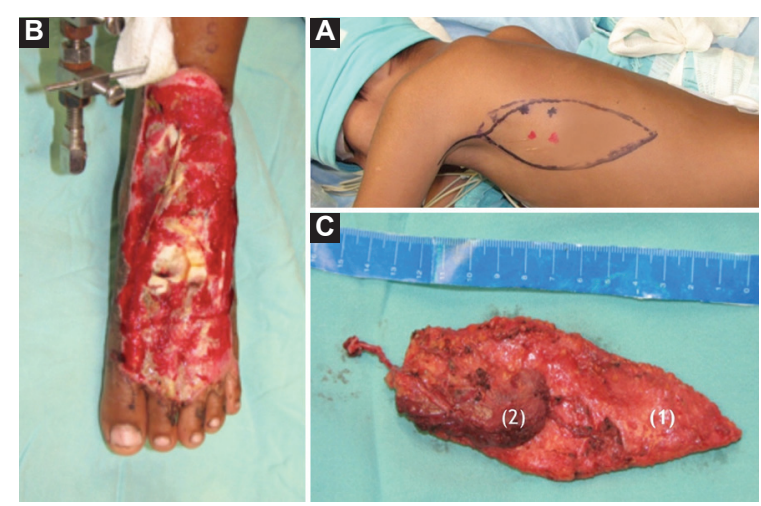

Figura 2. Marcaje, área cruenta y colgajo en un paciente varón de 8 años con una lesión en el dorso del pie izquierdo por un accidente automovilístico. A: identificación y marcaje mediante Doppler portátil de las arterias perforantes en el trayecto de la rama descendente de la arteria toracodorsal. B: área cruenta en el dorso del pie izquierdo, con pérdida de tendones extensores. C: colgajo con isla de piel (1) y una porción de músculo dorsal ancho (2).

tipo Likert ${ }^{29}$ de 4 puntos diseñada como protocolo de evaluación para este estudio ( 0 = cicatriz casi imperceptible, 1 = cicatriz poco evidente, 2 = cicatriz moderadamente evidente, 3 = cicatriz muy evidente), y la simetría corporal de la espalda y de la extremidad inferior $(0=$ excelente, 1 = buena, 2 = mala, 3 = muy mala).

\section{EVALUACIÓN FUNCIONAL}

La evaluación funcional se realizó al tercer mes del posoperatorio, en dos partes: una fue realizada por el padre o tutor, y otra por dos cirujanos por separado, uno que formaba parte del equipo de investigación y otro ciego a la investigación, por lo que se obtuvieron tres evaluaciones de cada paciente (39 evaluaciones en total). 
Tabla 1. Datos demográficos y complicaciones

\begin{tabular}{lcllll}
\hline Paciente & Edad (años) & Sexo & Causa de la lesión & Días hasta realizar el colgajo & Complicaciones \\
\hline 1 & 17 & M & Traumatismo & 6 & No \\
2 & 14 & M & Quemadura & 3 & No \\
3 & 15 & F & Traumatismo & 3 & No \\
4 & 10 & M & Resección oncológica & 1 & No \\
5 & 4 & M & Traumatismo & 4 & No \\
6 & 15 & F & Traumatismo & 2 & No \\
7 & 16 & M & Traumatismo & 15 & Necrosis parcial de la piel \\
8 & 9 & F & Traumatismo & 2 & No \\
9 & 14 & M & Traumatismo & 7 & No \\
10 & 12 & F & Resección oncológica & 1 & Pérdida parcial del colgajo \\
11 & 10 & M & Traumatismo & 3 & No \\
12 & 12 & F & Traumatismo & 4 & No \\
13 & 10 & M & Resección oncológica & 1 & Dehiscencia de 4 cm del sitio donador \\
\hline F: femenino; M: masculino. & & &
\end{tabular}

La valoración del padre o tutor, subjetiva, se refería a la movilidad del brazo y se pidió que fuera descrita en una escala tipo Likert ${ }^{29}$ diseñada como protocolo de evaluación para este estudio $(0=$ realiza todos los movimientos sin problema, 1 = realiza los movimientos con dificultad leve, 2 = realiza los movimientos con dificultad moderada a grave, $3=$ no puede realizar los movimientos). La evaluación por los cirujanos incluyó la capacidad para deambular (deambula sin ayuda, deambula con ayuda o no deambula) y la capacidad para realizar la extensión, la aducción y la rotación del hombro. Esta última evaluación se basó en la escala de fuerza muscular del Medical Research Council ${ }^{30}$ ( $\mathrm{M} 0$ = ninguna contracción, $\mathrm{M} 1$ = contracción débil que no desplaza la articulación, M2 = movimiento activo sin oposición de la gravedad, M3 = movimiento activo contra la fuerza de la gravedad, M4 = movimiento activo contra la fuerza de gravedad y la resistencia del examinador, M5 = fuerza normal).

Este estudio fue aprobado por el comité de ética e investigación del Hospital Universitario Dr. José Eleuterio González.

\section{Resultados}

Se incluyeron 14 pacientes en el periodo de estudio, de los cuales uno evolucionó con necrosis del colgajo (7.1\%), el cual se excluyó, quedando 13 pacientes para el análisis de los resultados, 8 de ellos de sexo masculino y 5 de sexo femenino, con una edad promedio de 12.1 años y un rango de 4 a 17 años.

Los 13 colgajos fueron de dorsal ancho preservadores de músculo y el seguimiento promedio fue de 6.3 meses, con un rango de 3.1 a 9.5 meses. La etiología más frecuente de las lesiones fue trauma en 9 pacientes (69\%); en 3 pacientes (23\%) fue secundario a una resección oncológica y en 1 paciente $(8 \%)$ fue por quemaduras. El tiempo transcurrido desde el momento de la lesión hasta el procedimiento quirúrgico fue en promedio de 4 días, con un rango de 1 a 15 días. En 3 pacientes (23\%) se presentaron complicaciones posoperatorias (Tabla 1).

En cuanto a la evaluación de la cicatriz (apariencia estética en la espalda), de las 39 evaluaciones, el $41 \%$ de los evaluadores refirieron una cicatriz casi imperceptible, el 38\% poco evidente, el $8 \%$ moderadamente evidente y el $13 \%$ muy evidente (Tabla 2). En la apariencia estética de la cicatriz en la extremidad inferior, los evaluadores refirieron también que la cicatriz era casi imperceptible en el $41 \%$, poco evidente en el $26 \%$, moderadamente evidente en el $15 \%$ y muy evidente en el 18\% (Tabla 3). En la evaluación de la apariencia estética de la simetría corporal de la espalda, de las 39 evaluaciones, la clasificaron como excelente el $67 \%$, buena el $15 \%$, mala el $10 \%$ y muy mala el $8 \%$ (Tabla 2). En la apariencia estética 
Tabla 2. Evaluación de la apariencia estética del sitio donador (espalda)

\begin{tabular}{|c|c|c|c|c|c|c|}
\hline \multirow[t]{2}{*}{ Paciente } & \multicolumn{3}{|c|}{ Cicatriz } & \multicolumn{3}{|c|}{ Simetría } \\
\hline & Padre & $\begin{array}{c}\text { Cirujano } \\
1\end{array}$ & $\begin{array}{c}\text { Cirujano } \\
2\end{array}$ & Padre & $\begin{array}{c}\text { Cirujano } \\
1\end{array}$ & $\begin{array}{c}\text { Cirujano } \\
2\end{array}$ \\
\hline 1 & 3 & 3 & 2 & 1 & 1 & 3 \\
\hline 2 & 1 & 1 & 1 & 0 & 0 & 2 \\
\hline 3 & 0 & 0 & 0 & 0 & 0 & 1 \\
\hline 4 & 1 & 1 & 0 & 0 & 0 & 0 \\
\hline 5 & 1 & 2 & 1 & 0 & 0 & 2 \\
\hline 6 & 0 & 0 & 0 & 0 & 0 & 0 \\
\hline 7 & 3 & 3 & 1 & 1 & 1 & 3 \\
\hline 8 & 0 & 0 & 0 & 0 & 0 & 1 \\
\hline 9 & 1 & 1 & 1 & 0 & 0 & 2 \\
\hline 10 & 1 & 0 & 0 & 0 & 0 & 0 \\
\hline 11 & 0 & 0 & 0 & 0 & 0 & 0 \\
\hline 12 & 1 & 1 & 0 & 0 & 0 & 2 \\
\hline 13 & 3 & 2 & 1 & 0 & 0 & 3 \\
\hline
\end{tabular}

Resultados de las 39 evaluaciones de la apariencia estética de la espalda basados en una escala tipo Likert. Cicatriz: $0=$ casi imperceptible $(16$ [41\%]), $1=$ poco evidente $(15[38 \%]), 2=$ moderadamente evidente $(3[8 \%]), 3=$ muy evidente $(5[13 \%])$. Simetría: $0=$ excelente $(26[67 \%]), 1=$ buena $(6[15 \%]), 2=$ mala $(4[10 \%]), 3=$ muy mala (3 [8\%]).
Tabla 3. Evaluación de la apariencia estética en el sitio receptor (extremidad inferior)

\begin{tabular}{|c|c|c|c|c|c|c|}
\hline \multirow[t]{2}{*}{ Paciente } & \multicolumn{3}{|c|}{ Cicatriz } & \multicolumn{3}{|c|}{ Simetría } \\
\hline & Padre & $\begin{array}{c}\text { Cirujano } \\
1\end{array}$ & $\begin{array}{c}\text { Cirujano } \\
2\end{array}$ & Padre & $\begin{array}{c}\text { Cirujano } \\
1\end{array}$ & $\begin{array}{c}\text { Cirujano } \\
2\end{array}$ \\
\hline 1 & 3 & 3 & 3 & 1 & 1 & 1 \\
\hline 2 & 2 & 2 & 2 & 0 & 0 & 0 \\
\hline 3 & 0 & 0 & 0 & 0 & 0 & 0 \\
\hline 4 & 1 & 1 & 1 & 0 & 0 & 1 \\
\hline 5 & 1 & 1 & 0 & 0 & 0 & 0 \\
\hline 6 & 0 & 0 & 0 & 0 & 0 & 0 \\
\hline 7 & 3 & 3 & 1 & 0 & 0 & 0 \\
\hline 8 & 0 & 0 & 0 & 0 & 0 & 0 \\
\hline 9 & 1 & 2 & 0 & 0 & 0 & 0 \\
\hline 10 & 0 & 0 & 0 & 0 & 0 & 0 \\
\hline 11 & 0 & 1 & 0 & 0 & 0 & 1 \\
\hline 12 & 2 & 2 & 1 & 0 & 0 & 0 \\
\hline 13 & 3 & 3 & 1 & 1 & 1 & 1 \\
\hline
\end{tabular}

Resultados de las 39 evaluaciones de la apariencia estética de la extremidad inferior basados en una escala tipo Likert. Cicatriz: $0=$ casi imperceptible (16 [41\%], $1=$ poco evidente $(10[26 \%], 2=$ moderadamente evidente $(6[15 \%]), 3=$ muy evidente $(7[18 \%])$. Simetría: $0=$ excelente $(31[79 \%]), 1=$ buena $(8[21 \%]), 2=$ mala $(0[0 \%])$ $3=$ muy mala $(0[0 \%])$. y la simetría de la extremidad inferior, se catalogó como simetría excelente el $79 \%$ y buena el $21 \%$ (Tabla 3).

En la valoración subjetiva de la movilidad del brazo, por parte del padre o tutor, consideraron que realizan todos los movimientos sin problema el $54 \%$ y con dificultad leve el $46 \%$.

En cuanto a la deambulación, 9 pacientes (69\%) la realizaron sin problema, 3 pacientes $(23 \%)$ la realizaron sin apoyo y 1 paciente (8\%) no pudo deambular debido a una rotura tendinosa que no se le había diagnosticado.

En las 39 evaluaciones de la fuerza muscular del hombro, se catalogó como M5 el $62 \%$ en la extensión, el $77 \%$ en la aducción y el $62 \%$ en la rotación interna (Tabla 4).

En la toma del colgajo, en 8 casos se incluyó una perforante y en 5 casos se incluyeron dos perforantes. En 10 de 13 colgajos se encontraron las perforantes en un diámetro de $3 \mathrm{~cm}$ sobre el marcaje mencionado. El tamaño de la isla de piel utilizada estuvo en un rango de $6 \mathrm{~cm}$ de ancho y hasta $25 \mathrm{~cm}$ de longitud. Se llevó a cabo el cierre primario del sitio donador en todos los pacientes. Se obtuvo la supervivencia del colgajo en 13 de los 14 casos (92.9\%). En el posoperatorio de los 13 pacientes se presentaron tres complicaciones (23\%): una pérdida parcial del colgajo que fue manejada con curaciones y epitelizó, en otro paciente el colgajo sufrió solo una pérdida parcial de piel y se resolvió con injerto de piel de espesor parcial, y una dehiscencia de $4 \mathrm{~cm}$ de la herida en el sitio donador en otro caso que se manejo con cierre terciario.

El contorno y la apariencia estética fueron satisfactorios en la mayoría de los pacientes, y tuvieron la capacidad de utilizar calzado sin problemas. Se conservó el pliegue axilar posterior en todos los casos. No se presentaron infecciones, seromas ni hematomas.

\section{Discusión}

Con la introducción del colgajo miocutáneo libre basado en perforantes, el concepto del músculo como elemento que proporciona vascularidad ya no es necesario. El empleo de este tipo de colgajo evita un grosor innecesario y preserva la función muscular. 
Tabla 4. Evaluación funcional del sitio donador de la extremidad superior (dorsal ancho residual)

\begin{tabular}{|c|c|c|c|c|c|c|}
\hline \multirow[t]{2}{*}{ Paciente } & \multicolumn{3}{|c|}{ Cirujano 1} & \multicolumn{3}{|c|}{ Cirujano 2} \\
\hline & $\begin{array}{l}\text { Extensión } \\
\text { del hombro }\end{array}$ & $\begin{array}{l}\text { Aducción } \\
\text { del hombro }\end{array}$ & $\begin{array}{c}\text { Rotación interna } \\
\text { del hombro }\end{array}$ & $\begin{array}{l}\text { Extensión del } \\
\text { hombro }\end{array}$ & $\begin{array}{l}\text { Aducción del } \\
\text { hombro }\end{array}$ & $\begin{array}{c}\text { Rotación interna del } \\
\text { hombro }\end{array}$ \\
\hline 1 & M5 & M5 & M5 & M5 & M5 & M5 \\
\hline 2 & M5 & M5 & M5 & M5 & M5 & M5 \\
\hline 3 & M4 & M4 & M4 & M4 & M4 & M4 \\
\hline 4 & M4 & M4 & M3 & M4 & M4 & M3 \\
\hline 5 & M5 & M5 & M5 & M5 & M5 & M5 \\
\hline 6 & M5 & M5 & M5 & M5 & M5 & M5 \\
\hline 7 & M5 & M5 & M5 & M5 & M5 & M5 \\
\hline 8 & M5 & M5 & M5 & M5 & M5 & M5 \\
\hline 9 & M5 & M5 & M5 & M5 & M5 & M5 \\
\hline 10 & M4 & M5 & M4 & M4 & M5 & M4 \\
\hline 11 & M4 & M4 & M4 & M4 & M4 & M4 \\
\hline 12 & M5 & M5 & M5 & M5 & M5 & M5 \\
\hline 13 & M4 & M5 & M4 & M4 & M5 & M4 \\
\hline MO & $0(0 \%)$ & $0(0 \%)$ & $0(0 \%)$ & $0(0 \%)$ & $0(0 \%)$ & $0(0 \%)$ \\
\hline M1 & $0(0 \%)$ & $0(0 \%)$ & $0(0 \%)$ & $0(0 \%)$ & $0(0 \%)$ & $0(0 \%)$ \\
\hline M2 & $0(0 \%)$ & $0(0 \%)$ & $0(0 \%)$ & $0(0 \%)$ & $0(0 \%)$ & $0(0 \%)$ \\
\hline M3 & $0(0 \%)$ & $0(0 \%)$ & $1(7 \%)$ & $0(0 \%)$ & $0(0 \%)$ & $1(7 \%)$ \\
\hline M4 & $5(38 \%)$ & $3(23 \%)$ & $4(31 \%)$ & $5(38 \%)$ & $3(23 \%)$ & $4(31 \%)$ \\
\hline M5 & $8(62 \%)$ & $10(77 \%)$ & $8(62 \%)$ & $8(62 \%)$ & $10(77 \%)$ & $8(62 \%)$ \\
\hline Total & $13(100 \%)$ & $13(100 \%)$ & $13(100 \%)$ & $13(100 \%)$ & $13(100 \%)$ & $13(100 \%)$ \\
\hline
\end{tabular}

Resultados de la evaluación de la fuerza muscular de la extremidad superior de acuerdo con la escala de fuerza muscular del Medical Research Council. M0 = ninguna contracción, M1 = contracción débil que no desplaza la articulación, M2 = movimiento activo sin oposición de la gravedad, M3 = movimiento activo contra la fuerza de la gravedad,

M4 = movimiento activo contra la fuerza de la gravedad y resistencia del examinador, M5 = fuerza normal.

Valoramos la estética en los sitios receptor y donador, parámetro considerado importante para el pronóstico del paciente. Además, en los pacientes con lesiones en el pie evaluamos la capacidad de utilizar calzado, aspecto no valorado en publicaciones previas y básico para la integración social.

Buntic, et al. ${ }^{22}$ reportaron el uso de colgajo dorsal ancho preservador de músculo en 13 pacientes, dos de ellos pediátricos. Los colgajos incluyeron la rama interna o medial de la arteria toracodorsal, tomando la parte superior, y reportaron buena simetría torácica y buen funcionamiento del dorsal ancho. Utilizaron el dolor persistente, la debilidad del sitio donador y la fatiga temprana a los movimientos relacionados con la utilización del dorsal ancho como parámetros para valorar la función muscular. En nuestro estudio no valoramos el parámetro del dolor en la región torácica, porque consideramos que es un factor sobre el sitio donador y no se correlaciona con el objetivo de nuestro estudio.

Schwabegger, et al. ${ }^{27}$ realizaron una evaluación subjetiva y objetiva únicamente del sitio donador, con un resultado excelente en la mayoría de los casos, identificando deficiencias en el contorno. La función motora solo la evaluaron con la maniobra de extender la articulación del hombro con la extremidad en extensión en contra de la mano del explorador. Esta publicación incluyó pacientes adultos y niños, y la reconstrucción de diversos defectos, pero no de la extremidad inferior. 
Nuestra evaluación motora consideró los tres movimientos de la articulación del hombro en los que participa el músculo dorsal ancho (extensión, aducción y rotación interna), y el hecho de incluir a dos cirujanos nos da una información más objetiva. La apreciación por parte del padre o tutor de la movilidad del hombro fue de gran importancia, ya que ellos valoran sus actividades diarias y es algo que no se puede ver en la consulta.

El régimen para anticoagulación no está establecido en los niños. Canales, et al. ${ }^{7}$ utilizaron dextrano de bajo peso molecular por 5 días y aspirina por 4 semanas. Nosotros, al igual que Van Beek, et al. ${ }^{31} \mathrm{y}$ Chiang, et al. ${ }^{3}$, utilizamos dextrano 40 por 4 días y aspirina de $100 \mathrm{mg}$ por 15 días.

En el presente estudio de 14 casos ocurrió la pérdida de un colgajo por trombosis venosa y hubo dos pacientes con pérdida parcial. En la serie de Canales, et al. ${ }^{7}$ hubo una pérdida de 13 colgajos en 106 pacientes. Seong, et al. ${ }^{32}$ reportan cuatro pacientes pediátricos con reconstrucción de defectos con colgajo perforante de la arteria toracodorsal sin pérdida de colgajos.

En un estudio sobre reconstrucción microquirúrgica con colgajos libres en niños, en los que utilizaron cinco colgajos de dorsal ancho, con sacrificio muscular importante, hubo una morbilidad del $35.3 \%{ }^{33}$.

Tanto el colgajo dorsal ancho preservador de músculo como el colgajo perforante de la arteria toracodorsal presentan mejores resultados funcionales y estéticos en el sitio donador que el colgajo libre de dorsal ancho. Sin embargo, el colgajo perforante de la arteria toracodorsal requiere una disección microquirúrgica más meticulosa de las perforantes, sobre todo en vasos tan pequeños como los de los niños, requiere mayor tiempo quirúrgico y tiene un mayor riesgo de pérdida del colgajo que con el colgajo dorsal ancho preservador de músculo ${ }^{23,25}$.

Se prefirió como donador el dorsal ancho contralateral al sitio de la extremidad inferior lesionada para poder trabajar en dos equipos. El grosor de la piel hasta la fascia profunda en la parte alta y lateral de la espalda es usualmente comparable con la parte distal de la extremidad inferior, como demuestra la simetría obtenida en el sitio receptor. La cicatriz resultante del área donadora fue de buenas características y además se oculta con la ropa.

Los pacientes incluidos en nuestro estudio representan la mayor cantidad de pacientes pediátricos con defectos en la extremidad inferior tratados con un colgajo de dorsal ancho preservador de músculo. Los resultados de nuestro estudio nos permiten proponer

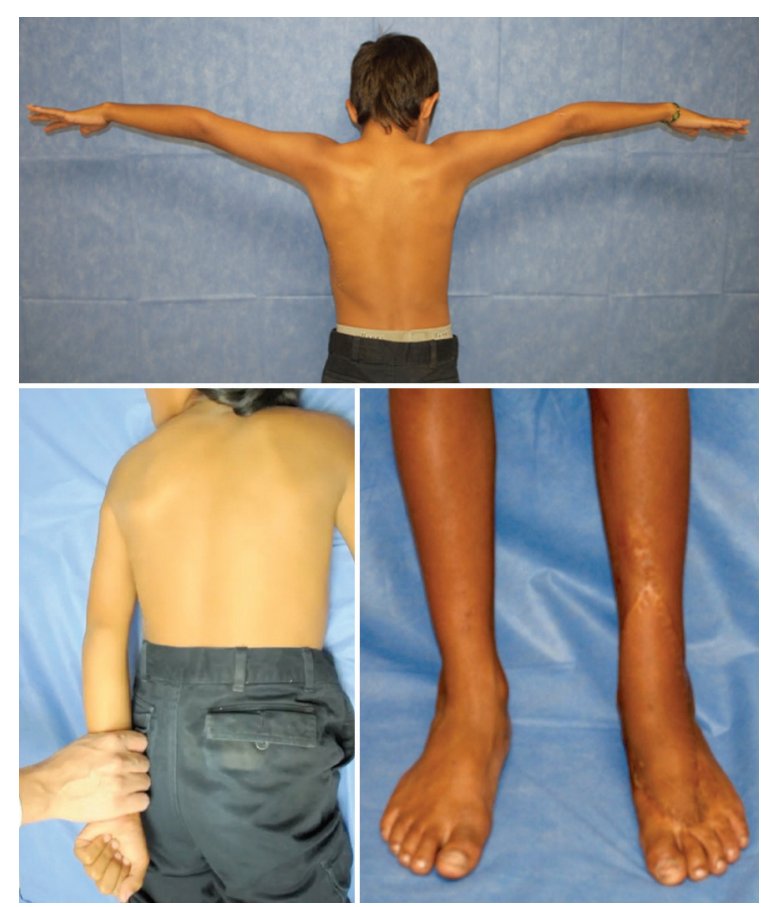

Figura 3. Seguimiento y valoración posoperatoria de un paciente varón de 8 años con un área cruenta en el dorso del pie izquierdo por un accidente automovilístico, al cual se le realizó colgajo dorsal ancho preservador de músculo. Adecuada apariencia estética de la cicatriz en la espalda y la extremidad inferior, simetría en espalda y fuerza muscular preservada.

otra opción para la reconstrucción de defectos tisulares importantes de la extremidad inferior en los niños.

La procuración del colgajo de dorsal ancho preservador de músculo es una técnica valiosa y logra un doble objetivo: preservar la función muscular y la apariencia estética del pliegue axilar posterior. Este colgajo, además, provee un pedículo largo y con una isla de piel delgada para reconstruir defectos en la extremidad inferior en los niños, dando como resultado la cobertura del defecto con un colgajo que asemeja los tejidos blandos a este nivel (Fig. 3).

\section{Conclusión}

El colgajo de dorsal ancho preservador de músculo tiene un grado de satisfacción muy elevado, con una excelente simetría y con preservación de la función, por lo que consideramos que es una técnica ideal para la reconstrucción de defectos en la extremidad inferior en los niños y proponemos que sea tomado en consideración como una opción reconstructiva en defectos tisulares importantes en la extremidad inferior en población pediátrica. 


\section{Responsabilidades éticas}

Protección de personas y animales. Los autores declaran que para esta investigación no se han realizado experimentos en seres humanos ni en animales.

Confidencialidad de los datos. Los autores declaran que han seguido los protocolos de su centro de trabajo sobre la publicación de datos de pacientes.

Derecho a la privacidad y consentimiento infor-

mado. Los autores han obtenido el consentimiento informado de los pacientes y/o sujetos referidos en el artículo. Este documento obra en poder del autor de correspondencia.

\section{Conflicto de intereses}

Los autores declaran no tener ningún conflicto de intereses.

\section{Bibliografía}

1. Rinker B, Valerio IL, Stewart DH, Pu LLQ, Vasconez HC. Microvascular free flap reconstruction in pediatric lower extremity trauma: A 10-Year review. Plast Reconstr Surg. 2005;115:1618-24.

2. Yücel A, Aydin Y, Yazar Ş, Altintaş F, Şenyuva C. Elective free-tissue transfer in pediatric patients. J Reconstr Microsurg. 2001;17:27-36.

3. Chiang YC, Jeng SF, Yeh MC, Liu YT, Chen HT, Wei FC. Free tissue transfer for leg reconstruction in children. Br J Plast Surg. 1997;50:335-42.

4. Shenaq SM, Dinh TA. Pediatric microsurgery: replantation, revascularization, and obstetric brachial plexus palsy. Clin Plast Surg. 1990;17:77-83

5. Devaraj VS, Kay SP, Batchelor AG, Yates A. Microvascular surgery in children. Br J Plast Surg. 1991;44:276-80.

6. Arnez ZM, Hanel DP. Free tissue transfer for reconstruction of traumatic limb injuries in children. Microsurgery. 1991;12:207-15.

7. Canales F, Lineaweaver WC, Furnas H, Whitney TM, Siko PP, Alpert BS, et al. Microvascular tissue transfer in paediatric patients: analysis of 106 cases. Br J Plast Surg. 1991;44:423-7.

8. Hallock GG. Efficacy of free flaps for pediatric trauma patients in an adult trauma center. J Reconstr Microsurg. 1995;11:169-74

9. Serletti JM, Schingo VA, Deuber MA, Carras AJ, Raul Herrera H, Reale VF. Free tissue transfer in pediatric patients. Ann Plast Surg. 1996;36:561-8.

10. Van Landuyt K, Hamdi M, Blondeel P, Tonnard P, Verpaele A, Monstrey S. Free perforator flaps in children. Plast Reconstr Surg. 2005;116:159-69.

11. Bouffaut AL, Hamel A, Guillard S, Pannier M, Duteille F. Use of muscle free flaps in lower limbs reconstruction in children. Ann Chir Plast Esthet. 2008;53:267-71.

12. Duteille F, Lim A, Dautel G. Free flap coverage of upper and lower limb tissue defects in children: a series of 22 patients. Ann Plast Surg. 2003;50:344-9.
13. Kim JT, Kim SW, Youn S, Kim YH. What is the ideal free flap for soft tissue reconstruction? A ten-year experience of microsurgical reconstruction using 334 latissimus dorsi flaps from a universal donor site. Ann Plast Surg. 2015;75:49-54

14. Spear SL, Hess CL. A review of the biomechanical and functional changes in the shoulder following transfer of the latissimus dorsi muscles. Plast Reconstr Surg. 2005;115:2070-3.

15. Ismaïl M, Boucher F, Chauvel-Picard J, Shipkov H, Braye F, Mojallal A Sequelae after harvesting latissimus dorsi flap and derivates - Review. Ann Chir Plast Esthet. 2014;59:348-54.

16. Lee KT, Mun GH. A systematic review of functional donor-site morbidity after latissimus dorsi muscle transfer. Plast Reconstr Surg. 2014;134:303-14

17. Berger A, Bargmann HJ. Aesthetic aspects in reconstructive microsurgery. Aesth. Plast Surg. 1989;13:115-20.

18. Kim DH, Glazer PA. Progression of idiopathic thoracolumbar scoliosis after breast reconstruction with a latissimus dorsi flap: a case report. Spine (Phila Pa 1976). 2000;25:622-5.

19. Chen S-L. Thoracodorsal artery perforator flap for extremity reconstruction. Semin Plast Surg. 2006;20:112-20.

20. Saint-Cyr M, Schaverien MV, Rohrich RJ. Perforator flaps: history, controversies, physiology, anatomy, and use in reconstruction. Plast Reconstr Surg. 2009;123:132-45.

21. Schwabegger AH, Harpf $\mathrm{C}$, Rainer C. Muscle-sparing latissimus dorsi myocutaneous flap with maintenance of muscle innervation, function, and aesthetic appearance of the donor site. Plast Reconstr Surg. 2003;111:1407-11

22. Buntic RF, Horton KM, Brooks D, Lee CK. The free partial superior latissimus muscle flap: preservation of donor-site form and function. Plast Reconstr Surg. 2008;121:1659-63.

23. Brooks Darrell BRF. Partial muscle harvest: our first 100 cases attempting to preserve form and function at the donor site. Microsurgery. 2008;(28):606-11.

24. Ortiz CL, Mendoza MM, Sempere LN, Sanz JS, Torres AN, Barraquer EL. Versatility of the pedicled thoracodorsal artery perforator (TDAP) flap in soft tissue reconstruction. Ann Plast Surg. 2007:58:315-20.

25. Colohan S, Wong C, Lakhiani C, Cheng A, Maia M, Arbique G, et al. The free descending branch muscle-sparing latissimus dorsi flap: vascular anatomy and clinical applications. Plast Reconstr Surg. 2012;130:776-87.

26. Namdar T, Stollwerck PL, Stang F, Lange T, Mailander P, Siemers F. Latissmus dorsi muscle flap for lower extremity reconstruction in children. Microsurgery. 2010;(30):504-6.

27. Schwabegger A, Harpf C, Rainer C. Muscle-sparing latissimus dorsi myocutaneous flap with maintenance of muscle innervation, function, and aesthetic appearance of the donor site. Plast Reconstr Surg. 2003:111:1407-11.

28. Château J, Boucher F, Braye F, Erhard L, Mojallal A. Reconstruction of soft tissue defects of the distal third of the arm using a muscle-sparing latissimus dorsi musculocutaneous flap. Hand Surg Rehabil. 2018:37:16-9.

29. Matas A. Diseño del formato de escalas tipo Likert: un estado de la cuestión. Rev Electron Investig Educ. 2018;20:38-47.

30. James MA. Use of the Medical Research Council Muscle Strength Grading System in the Upper Extremity. J Hand Surg Am. 2007;32:154-6.

31. Van Beek AL, Wavak PW, Zook EG. Microvascular surgery in young children. Plast Reconstr Surg. 1979:63:457-62.

32. Seong EK, In HR, Bo GS, Chung KC. Use of thoracodorsal artery perforator flap for soft tissue reconstruction in children. Ann Plast Surg. 2006;56:451-4.

33. Organek AJ, Klebuc MJ, Zuker RM. Indications and outcomes of free tissue transfer to the lower extremity in children: review. J Reconstr Microsurg. 2006;22:173-81. 